\title{
Anti-IL-20 monoclonal antibody suppresses hepatocellular carcinoma progression
}

\author{
WEN-ZHOU DING $^{1 *}$, GUO-YONG HAN ${ }^{2 *}$, HUI-HAN JIN ${ }^{1 *}$, CHUAN-FEI ZHAN $^{2}$, YUAN JI $^{1}$ and XIN-LI HUANG ${ }^{3}$ \\ ${ }^{1}$ Department of Hepatobiliary Surgery, Nanjing Medical University Affiliated Wuxi Second Hospital, Wuxi, Jiangsu 214002; \\ ${ }^{2}$ Key Laboratory of Living Donor Liver Transplantation, National Health and Family Planning Commission, \\ Department of Liver Surgery, The First Affiliated Hospital of Nanjing Medical University, Nanjing, Jiangsu 210029; \\ ${ }^{3}$ Department of Hepatobiliary Surgery, Nanjing Drum Tower Hospital, The Affiliated Hospital of \\ Nanjing University Medical School, Nanjing, Jiangsu 210008, P.R. China
}

Received October 21, 2017; Accepted August 30, 2018

DOI: $10.3892 / \mathrm{ol} .2018 .9402$

\begin{abstract}
Interleukin(IL)-20 is a member of the IL-10 family of cytokines, which has been reported to participate in autoimmune inflammatory diseases. However, the potential role of IL-20 in hepatocellular carcinoma (HCC) progression has not yet been investigated. In the present study, it was observed that IL-20 mRNA and protein levels were markedly increased in the HCC tissues examined via reverse transcription-quantitative polymerase chain reaction and immunohistochemical staining. In addition, IL-20 expression was significantly associated with tumor size, metastasis, TNM stage and poor prognosis in patients with HCC. Mouse recombinant IL-20 (mIL-20) enhanced liver cancer cell proliferation, migration and invasion in vitro, while the anti-IL-20 monoclonal antibody (mAb) attenuated the effect of mIL-20, inhibiting cancer cell migration and invasion in vitro and suppressing cell growth in vitro and in vivo. This was detected by Cell Counting Kit- 8 , colony formation, Transwell assays and a xenograft tumor nude mouse model. Western blotting revealed that IL-20 promoted HCC progression through inducing transforming growth factor- $\beta$ and matrix metalloproteinase 9 expression and enhancing the phosphorylation of Jun N-terminal kinase and signal transducer and activator of transcription 3 . The results of the present study indicated that IL-20 promotes HCC development. In addition, anti-IL-20 mAb may attenuate the effect of IL-20 and suppress liver tumorigenesis in vitro and
\end{abstract}

Correspondence to: Dr Xin-Li Huang, Department of Hepatobiliary Surgery, Nanjing Drum Tower Hospital, The Affiliated Hospital of Nanjing University Medical School, 321 Zhongshan Road, Nanjing, Jiangsu 210008, P.R. China

E-mail: huangxinli@njmu.edu.cn

*Contributed equally

Key words: interleukin-20, anti-interleukin-20 monoclonal antibody, hepatocellular carcinoma, proliferation, metastasis in vivo, indicating that anti-IL-20 mAbs may potentially serve as effective therapeutic agents for HCC.

\section{Introduction}

Hepatocellular carcinoma (HCC) is one of the most common cancer types worldwide and the third leading cause of tumor-related deaths $(1,2)$. While significant progress has been achieved in surgical resection, liver transplantation and interventional therapy in recent years, the overall survival of patients with HCC remains unsatisfactory due to late diagnosis, early metastasis and a high rate of recurrence. Therefore, clarification of the mechanisms underlying HCC progression is essential to developing effective therapeutic strategies.

Interleukin (IL) 20 is a member of the IL-10 family of cytokines, which includes IL-10, IL-19, IL-22, IL-24 and IL-26 $(3,4)$. IL-20 shares $18-25 \%$ amino acid sequence identity with IL-10 (5). IL-20 acts by activating a heterodimer receptor complex of IL-20 receptor A/IL-20 receptor $B$ (IL-20RA/IL-20RB) or IL-22 receptor A/IL-20 receptor B (IL-22RA/IL-20RB) (6). Earlier studies have reported a close association of IL-20 with inflammatory diseases, such as psoriasis, rheumatoid arthritis and atherosclerosis $(7,8)$, and more recent investigations suggested an important role of IL-20 in tumor progression. Hsu et al (9), demonstrated that IL-20 accelerates breast cancer cell proliferation and migration, and enhances tumor progression through upregulating matrix metalloproteinase (MMP)-9, MMP-12, cathepsin K and cathepsin G. Moreover, the anti-IL-20 monoclonal antibody (mAb) 7E suppressed breast tumor growth in vivo. In bladder cancer, IL-20 was shown to promote cell migration and invasion via ERK1/2-dependent MMP-9 expression, and knockdown of IL-20R1 induced significant suppression of migration, invasion, ERK1/2 activation and NF- $\kappa \mathrm{B}$-mediated MMP-9 expression induced by IL-20 (10).

The liver is a potential target organ for IL-19, IL-20 and IL-24. IL-20 induces rapid tyrosine phosphorylation of the signal transducer and activator of transcription 3 (STAT3) by interacting with IL-20R heterodimers (type I or type II). In a previous study, STAT3 phosphorylation was enhanced upon treatment of cultured murine hepatocytes with IL-20 (11). IL-20 
is highly expressed in liver tissue under conditions of injury, such as fibrosis, cirrhosis and HCC. IL-20 activates hepatic stellate cells, upregulates TGF- $\beta 1$ expression and promotes the occurrence and progression of liver fibrosis. mAbs against IL-20 or IL-20R can attenuate hepatocyte damage caused by $\mathrm{CCl}_{4}$ and protect IL-20R1-deficient mice from short-term and long-term liver injury (12). These collective findings clearly support the involvement of IL-20 in liver disease progression. However, the specific functions and mechanisms of action of IL-20 in HCC require further exploration.

\section{Materials and methods}

Tissue specimens. Tumor tissue samples were obtained from 64 patients with HCC. Adjacent non-tumorous tissues were obtained from $2 \mathrm{~cm}$ away from the edge of the tumors. All patients underwent curative resection of HCC at the First Affiliated Hospital of Nanjing Medical University (Nanjing, China) between December 2010 and January 2012. The tissue samples were confirmed by pathological examination and immediately stored in liquid nitrogen after surgery. Written informed consent was obtained from individual patients prior to surgery. The study was approved by the Ethics Committee of the First Affiliated Hospital of Nanjing Medical University.

Cell lines and cultures. The mouse liver cancer cell line Hep1-6 was purchased from the Chinese Academy of Sciences Cell Bank (Shanghai, China). Cells were cultured in RPMI-1640 medium (Invitrogen; Thermo Fisher Scientific, Inc., Waltham, MA, USA) supplemented with $10 \%$ fetal bovine serum (FBS; Gibco; Thermo Fisher Scientific, Inc.) and 100 units/ml penicillin-streptomycin (Invitrogen; Thermo Fisher Scientific, Inc.), and maintained at $37^{\circ} \mathrm{C}$ in a humidified incubator with $5 \% \mathrm{CO}_{2}$.

RNA isolation and reverse transcription-quantitative polymerase chain reaction $(R T-q P C R)$. TRIzol reagent (Takara Biotechnology Co., Ltd., Dalian, China) was used, according to the manufacturer's protocol, to extract total RNA. The quality and concentration of RNA was determined using a Nanodrop ${ }^{\mathrm{TM}}$ 2000 spectrophotometer (NanoDrop Technologies; Thermo Fisher Scientific, Inc.). RT-qPCR was performed using SYBR Premix ExTaq (Takara Biotechnology, Co., Ltd.) with the ABI Prism 7900 HT Thermocycler (Applied Biosystems; Thermo Fisher Scientific, Inc.). $\beta$-actin was employed as the internal control for mRNA quantification. The primers used in the present study were as follows: IL-20 forward, 5'-ATG AAAGCCTCTAGTCTTGCCT-3' and reverse, 5'-GCCCCG TATCTCAGAAAATCC-3'; $\beta$-actin forward, 5'-AGAGCC TCGCCTTTGCCGATCC-3' and reverse, 5'-CTGGGCCTC GTCGCCCACATA-3'. The relative expression ratio of IL-20 in paired tumor and adjacent tissues was calculated using the $2^{-\Delta \Delta \mathrm{Cq}}$ method (13).

Western blot analysis. Specimens were lysed on ice in lysis buffer $50 \mathrm{mM}$ Tris- $\mathrm{Cl} \mathrm{pH} 7.4,150 \mathrm{mM} \mathrm{NaCl}, 0.5 \%$ sodium deoxycholate, $1 \%$ ethyl phenyl polyethylene glycol, $0.1 \%$ sodium dodecyl sulfonate (SDS), $1 \mathrm{mM}$ ethylenediaminetetraacetic acid (EDTA), $100 \mathrm{mM} \mathrm{NaF}, 1 \mathrm{mM} \mathrm{Na} \mathrm{VO}_{4}$, $1 \mathrm{mM}$ phenylmethanesulfonyl fluoride (PMSF), and $2 \mu \mathrm{g} / \mathrm{ml}$ aprotinin], and the total protein contents were determined with the Bradford method. Protein samples $(20 \mu \mathrm{g})$ were separated via 10\% SDS-polyacrylamide gel electrophoresis and transferred to polyvinylidene difluoride membranes. Membranes were blocked with $5 \%$ bull serum albumin in Tris-buffered saline with Tween (TBST) at room temperature for $2 \mathrm{~h}$, and then incubated with primary antibodies overnight at $4^{\circ} \mathrm{C}$. After washing with TBST three times for $15 \mathrm{~min}$ each, the membranes were incubated with the appropriate horseradish peroxidase-conjugated secondary antibodies for $2 \mathrm{~h}$ at room temperature. TBST wash steps were performed as described previously, and proteins were visualized using an enhanced chemiluminescence detection kit according to the manufacturer's recommendations (Beyotime Institute of Biotechnology, Haimen, China). Primary antibodies used in the present study included the following: Rabbit anti-IL-20 $\mathrm{mAb}$ (dilution, 1:150; R\&D Systems, Inc., Minneapolis, MN, USA), rabbit anti-MMP-9 polyclonal antibody (dilution, 1:1,000; Abcam, Cambridge, MA, USA), rabbit anti-p-STAT3 polyclonal antibody (dilution, 1:1,000), rabbit anti-p-JNK polyclonal antibody (dilution, 1:1,000), rabbit anti-TGF- $\beta$ polyclonal antibody (dilution, 1:1,000), and rabbit anti-GAPDH polyclonal antibody (dilution, 1:1,000) (all from Cell Signaling Technology, Inc., Danvers, MA, USA). The protein levels were calculated relative to the level of GAPDH.

Immunohistochemical staining. All tissues were paraffin-embedded and obtained from the Department of Pathology of The First Affiliated Hospital of Nanjing Medical University. Paraffin-embedded tissues were cut into $4-\mu \mathrm{m}$-thick sections, and incubated with the rabbit anti-IL-20 polyclonal antibody (dilution, 1:100; LifeSpan BioSciences, Inc., Seattle, WA, USA) overnight at $4^{\circ} \mathrm{C}$. SP-9000 Histostain ${ }^{\mathrm{TM}}$-Plus kits (ZSGB-Bio, Beijing, China) were used according to the manufacturer's protocols. Scoring was determined according to the intensity of staining in the cell cytoplasm: 0 , no staining; 1 , weak staining; 2 , moderate staining; and 3 , strong staining.

Cell proliferation assay. Hep1-6 cells were seeded into 96-well plates in $100 \mu \mathrm{l}$ complete medium, at a density of 2,000-5,000 cells/well. Mouse recombinant IL-20 (mIL-20) $(300 \mathrm{ng} / \mathrm{ml}$; R\&D Systems), anti-IL-20 mAb (3 mg/ml) or mIL-20 plus anti-IL-20 mAb (dilution, 1:10) was added to the culture system. A Cell Counting Kit-8 (CCK-8; Dojindo Molecular Technologies, Inc., Kumamoto, Japan) was used, according to the manufacturer's instructions, to measure the cell viability. Each experiment was repeated at least three times.

Colony formation assay. Hep1-6 cells were plated into $6-\mathrm{cm}$ dishes at a density of 400 cells/dish and incubated for $\sim 2$ weeks. The treatments [mIL-20 (300 ng/ml), anti-IL-20 mAb (3 mg/ml) or mIL-20 plus anti-IL-20 mAb (1:10)] were added to the culture system. The medium was changed every 3 days. Colonies were washed twice with phosphate-buffered saline (PBS; Gibco; Thermo Fisher Scientific, Inc.), stained with crystal violet (Beyotime Institute of Biotechnology), and images were obtained. Colonies of $>2 \mathrm{~mm}$ were counted, and used as indicators of the proliferation of single cells. The mean number of colonies per well was averaged from three wells for each experiment, and each experiment was repeated at least three times. 
Cell migration and invasion assays. The migration and invasion assays were performed using Transwell chambers (Corning Incorporated, Corning, NY, USA) with $8-\mu \mathrm{m}$ pore size. For the migration assay, $2 \times 10^{4}$ cells in $250 \mu 1$ RPMI-1640 medium containing $0.2 \%$ FBS were seeded into the upper chamber of each well. mIL-20 (300 ng/ml), anti-IL-20 mAb (3 $\mathrm{mg} / \mathrm{ml})$ or $\mathrm{mIL}-20$ plus anti-IL-20 mAb (1:10) was added to the upper chambers. The lower chambers were filled with $500 \mu 1$ RPMI-1640 medium containing 10\% FBS. Cells were incubated in $5 \% \mathrm{CO}_{2}$ at $37^{\circ} \mathrm{C}$ for $24 \mathrm{~h}$. Subsequently, the cells on the upper surface of the chamber were removed using a cotton tip, and the wells were fixed in methanol for $20 \mathrm{~min}$, then crystal violet was used to stain the migrated cells. Images from five random fields were captured for quantification and analysis. Three identical replicates were tested. For invasion, the upper chambers were coated with $100 \mu$ l Matrigel (dilution, 1:8; BD Biosciences, Franklin Lakes, NJ, USA) prior to seeding of the cells. The other steps were the same as for the migration assay.

In vivo tumorigenicity assay. Male nude mice, aged 4-6 weeks, were housed under specific pathogen-free conditions and cared for according to the institutional guidelines for animal care. All animal experiments met the National Institutes of Health guidelines and were approved by the Committee on the Ethics of Animal Experiments of Nanjing Medical University. In total, $5 \times 10^{6}$ cells were injected subcutaneously into the opposite inguen in the same mice. Animals were randomly assigned to three groups ( $n=3$ per group) and treated with PBS, membrane immunoglobulin G (mIgG) or anti-IL-20 $\mathrm{mAb}(5 \mathrm{mg} / \mathrm{kg}$; subcutaneous injection) every 5 days for the duration of the experiment. Mice were monitored every 5 days and sacrificed 35 days after injection. Tumor sizes, weights and volumes were determined for further analysis. Levels of related proteins were examined via western blotting, according to the aforementioned protocols.

Statistical analyses. Statistical analyses were performed using GraphPad Prism v.5.0 (GraphPad Software, La Jolla, CA, USA) software and SPSS v.18.0 (SPSS, Inc., Chicago, IL, USA). Quantitative data were presented as the mean \pm standard error of mean. Differences between two groups were evaluated using the two-tailed Student's t-test. The chi-square test was applied to analyze the clinical parameters. Kaplan-Meier analysis was applied to evaluate the prognosis. One-way analysis of variance with a non-parametric Kruskal-Wallis test were used to perform multiple comparisons. $\mathrm{P}<0.05$ was considered to indicate a statistically significant difference.

\section{Results}

$I L-20$ is overexpressed in HCC. We detected the mRNA expression of IL-20 in 64 pairs of HCC and adjacent non-tumorous tissues via RT-qPCR. As shown in Fig. 1A, IL-20 expression was significantly upregulated in the majority of primary $\mathrm{HCC}$ (53\%; 34/64) compared with in the corresponding non-tumor region $(\mathrm{P}<0.01)$. Immunohistochemical analysis confirmed a similar trend in the corresponding protein level. As shown in Fig. 1B, in the same 64 matched samples, 26/64 (40.6\%) of the cancerous specimens showed no or weak-positive $(-/+)$
Table I. Patient characteristics.

\begin{tabular}{|c|c|c|c|}
\hline Variables & $\begin{array}{l}\text { IL-20 high } \\
\text { (n) }\end{array}$ & $\begin{array}{c}\text { IL-20 low } \\
\text { (n) }\end{array}$ & P-value \\
\hline \multicolumn{4}{|c|}{ Age (years) } \\
\hline$\geq 55$ & 20 & 15 & \multirow[t]{2}{*}{0.4792} \\
\hline$<55$ & 14 & 15 & \\
\hline \multicolumn{4}{|l|}{ Gender } \\
\hline Male & 23 & 16 & \multirow[t]{2}{*}{0.2415} \\
\hline Female & 11 & 14 & \\
\hline \multicolumn{4}{|c|}{ AFP (ng/ml) } \\
\hline$\geq 400$ & 19 & 12 & \multirow[t]{2}{*}{0.2045} \\
\hline$<400$ & 15 & 18 & \\
\hline \multicolumn{4}{|c|}{ Liver cirrhosis } \\
\hline Yes & 27 & 15 & \multirow[t]{2}{*}{0.0134} \\
\hline No & 7 & 15 & \\
\hline \multicolumn{4}{|c|}{ Tumor size $(\mathrm{cm})$} \\
\hline$\geq 5$ & 25 & 10 & \multirow[t]{2}{*}{0.0013} \\
\hline$<5$ & 9 & 20 & \\
\hline \multicolumn{4}{|c|}{ Vascular invasion } \\
\hline Yes & 19 & 8 & \multirow[t]{2}{*}{0.0182} \\
\hline No & 15 & 22 & \\
\hline \multicolumn{4}{|c|}{ TNM stage } \\
\hline I-II & 6 & 14 & \multirow[t]{2}{*}{0.0332} \\
\hline III-IV & 28 & 20 & \\
\hline
\end{tabular}

IL, interleukin; TNM, tumor-node-metastasis; AFP, $\alpha$-fetoprotein.

staining, whereas $40 / 64(62.5 \%)$ of the non-tumor tissues showed no or weak-positive (-/+) staining.

Expression of IL-20 is significantly associated with poor prognosis in patients with HCC. We collected data from 64 patients with $\mathrm{HCC}$ and examined the associations of clinical parameters with IL-20 expression patterns. As shown in Table I, IL-20 expression was strongly associated with adverse clinicopathological prognosticators, such as liver cirrhosis $(\mathrm{P}=0.0134)$, tumor size $(\mathrm{P}=0.0013)$, vascular invasion $(\mathrm{P}=0.0182)$ and $\mathrm{TNM}$ stage $(\mathrm{P}=0.0332)$. However, we observed no significant correlations between IL-20 expression and patient age, patient sex or $\alpha$-fetoprotein level. Kaplan-Meier analysis was applied to evaluate the association between IL-20 expression and prognosis. Notably, patients with high IL-20 expression had poorer overall survival than those with low IL-20 expression $(\mathrm{P}<0.01$; Fig. 1C). Based on these findings, we hypothesize that IL-20 expression is frequently upregulated in HCC tissues and is associated with poor patient survival.

Anti-IL-20 mAb inhibits proliferation and colony formation of Hep1-6 cells. To clarify the precise role of anti-IL-20 mAb in liver tumor cell growth, mIL-20, anti-IL-20 mAb, mIL-20 plus anti-IL-20 mAb or PBS (control) were added into the cell culture medium. As shown by the CCK-8 assays (Fig. 2A), compared 
A
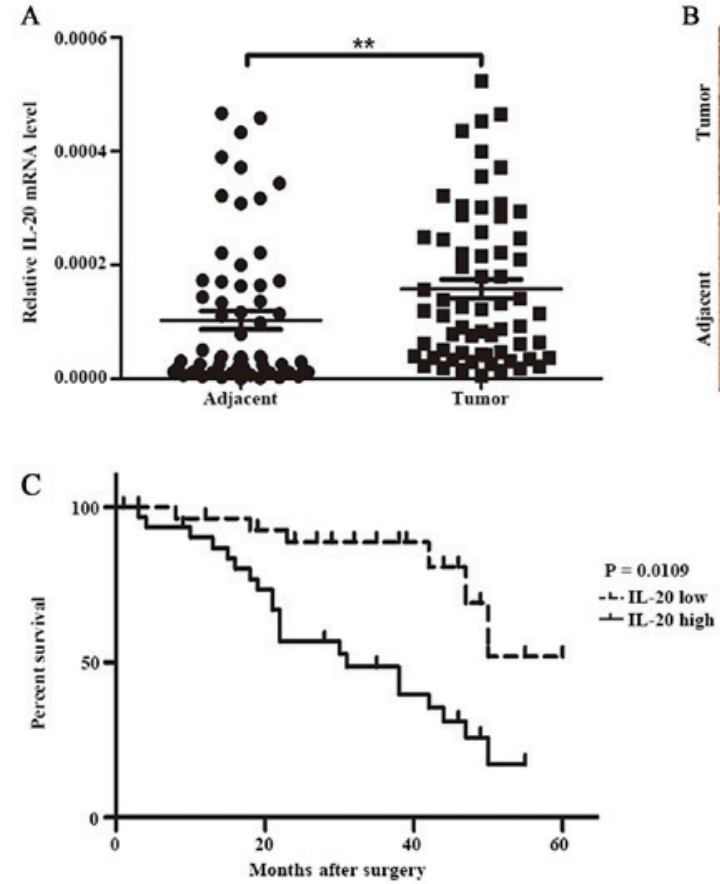

B
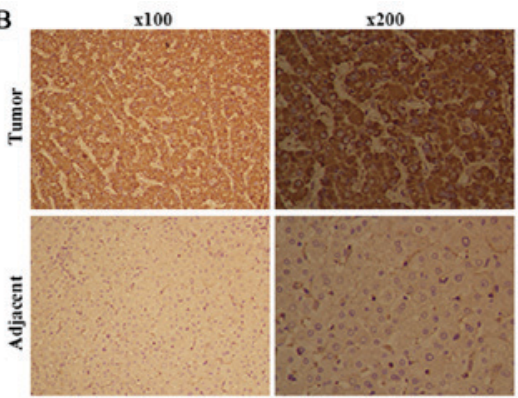

Figure 1. Expression of IL-20 in HCC and adjacent non-cancerous tissues. (A) IL-20 mRNA levels were examined by reverse transcription-quantitative polymerase chain reaction. ${ }^{* *} \mathrm{P}<0.01$, as indicated. (B) Protein levels of IL-20 were confirmed via immunohistochemistry. Magnification, x100 and x200 as indicated. (C) Association between IL-20 expression and overall survival time. IL, interleukin; HCC, hepatocellular carcinoma.
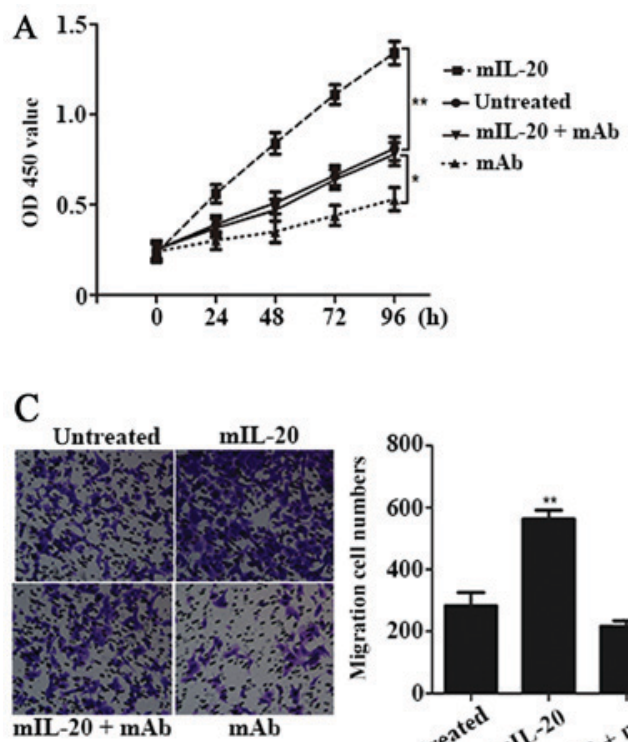

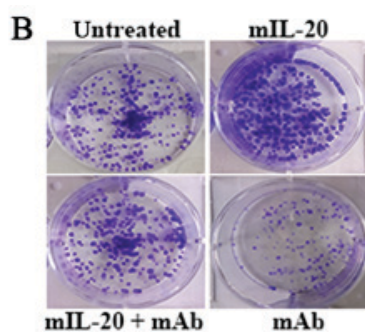

$\mathrm{D}$
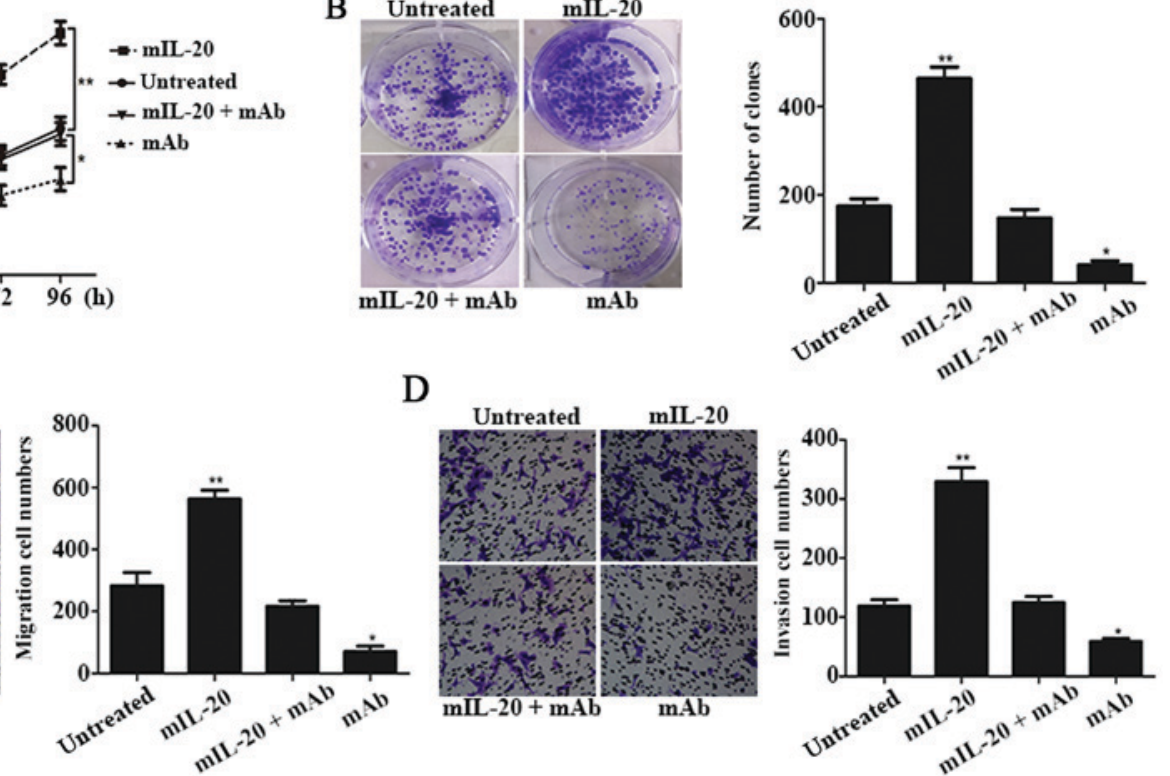

Figure 2. Growth, migration and invasion of Hep1-6 cells. (A) A Cell Counting Kit-8 assay was used to evaluate Hep1-6 proliferation. (B) A plate colony formation assay was used to evaluate Hep1-6 clonogenic ability. Transwell assays were performed to assess the (C) migration and (D) invasion of Hep1-6 cells (magnification, $\mathrm{x} 100$ ). The four groups were treated with mIL-20, anti-IL-20 mAb, mIL-20 plus mAb or PBS (untreated control). ${ }^{*} \mathrm{P}<0.05$ and ${ }^{* * *} \mathrm{P}<0.01$ vs. the untreated group. IL, interleukin; mAb, monoclonal antibody; mIL-20, mouse recombinant IL-20; OD, optical density.

with the control group, mIL-20 promoted cell proliferation $(\mathrm{P}<0.01)$, whereas anti-IL-20 mAb attenuated the effect of mIL-20 ( $\mathrm{P}>0.05$ vs. control), and inhibited Hep1-6 cell proliferation when applied alone ( $\mathrm{P}<0.05$ vs. control). As shown in Fig. 2B, colony formation in the presence of mIL-20 was significantly higher than that in the control group $(\mathrm{P}<0.01)$, while colony formation in the anti-IL-20 mAb group was inhibited $(\mathrm{P}<0.05$ vs. control). Furthermore, the effect of mIL-20 was counteracted by the anti-IL-20 specific $\mathrm{mAb}$ ( $\mathrm{P}>0.05$ vs. control).

Anti-IL-20 mAb impairs the invasion and migration of Hepl-6 cells. Transwell chamber assays were employed to evaluate the invasive and migratory abilities of Hep1-6 cells treated with mIL-20, anti-IL-20 mAb, mIL-20 plus anti-IL-20 mAb, 

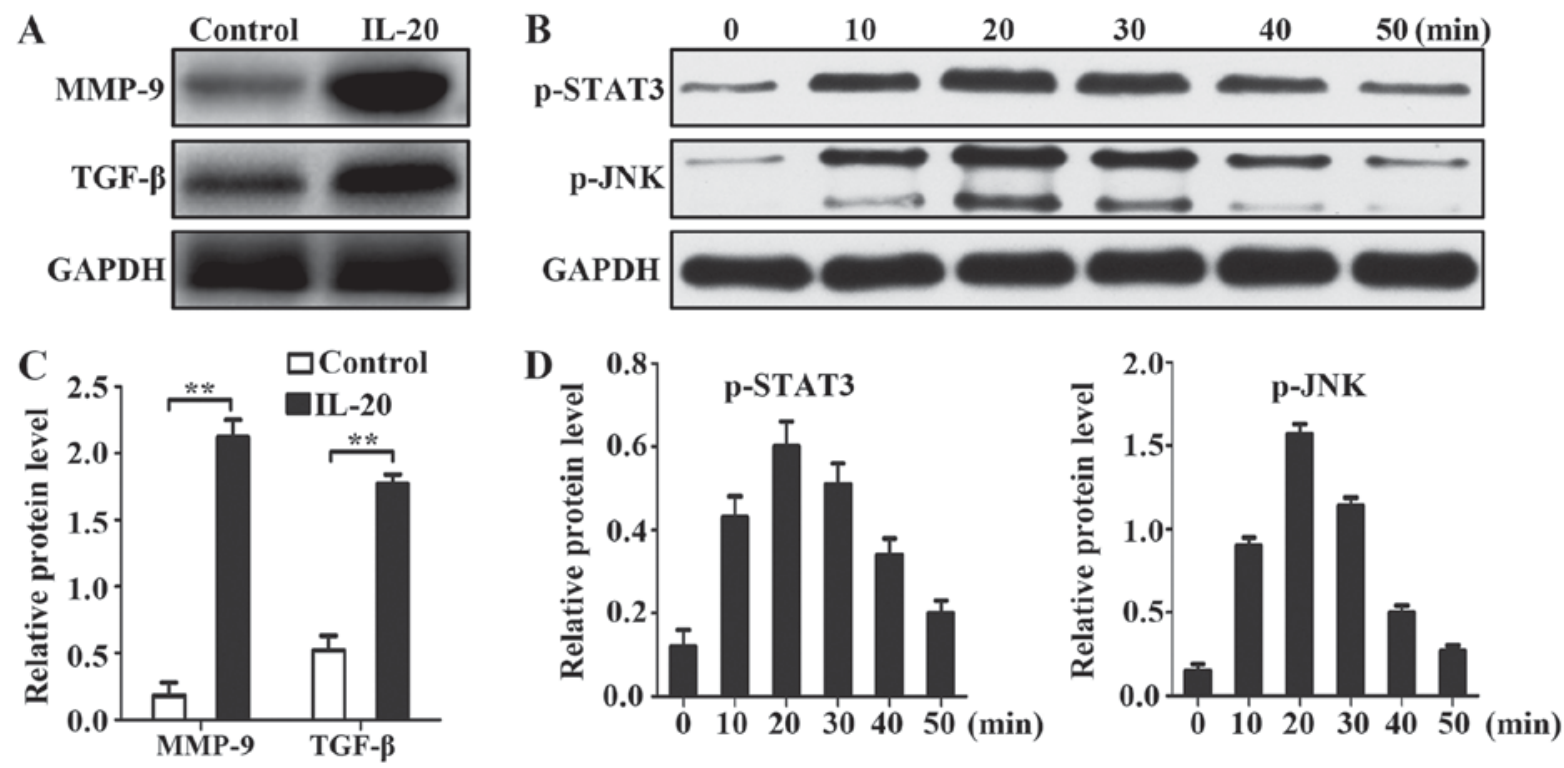

Figure 3. Western blot analysis of pathway-associated proteins. (A) Western blot analysis of MMP-9 and TGF- $\beta$ expression levels. (B) Detection of p-JNK and p-STAT3 for the indicated time periods using GAPDH as the internal control. (C) Gray scale analysis of MMP-9 and TGF- $\beta$ levels shown in panel A. ** P<0.01, as indicated. (D) Gray scale analysis of p-JNK and p-STAT3 levels shown in panel B. IL, interleukin; control, cell culture medium with PBS; p-, phosphorylated; MMP, matrix metalloproteinase; TGF, tumor growth factor; STAT, signal transducer and activator of transcription; JNK, Jun N-terminal kinase.

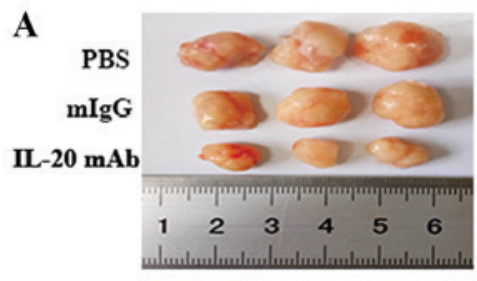

D

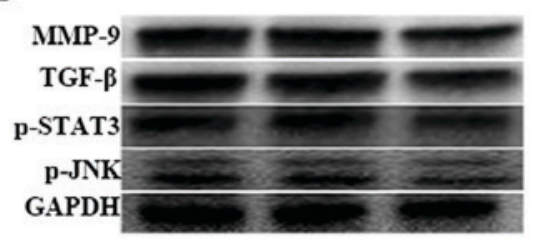

B
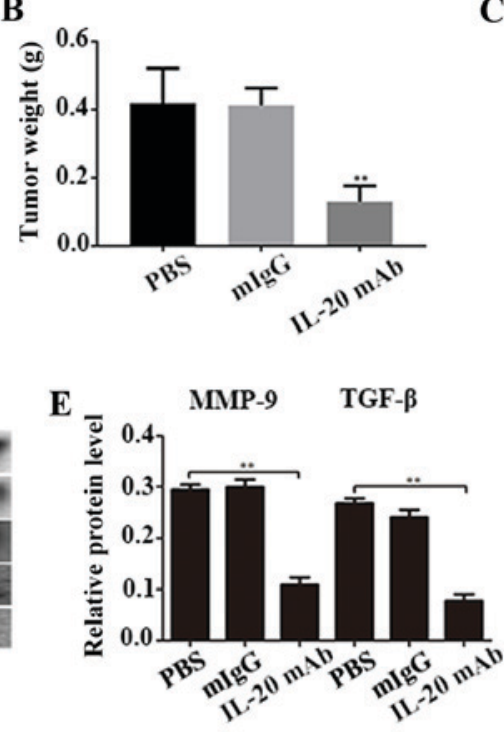

C
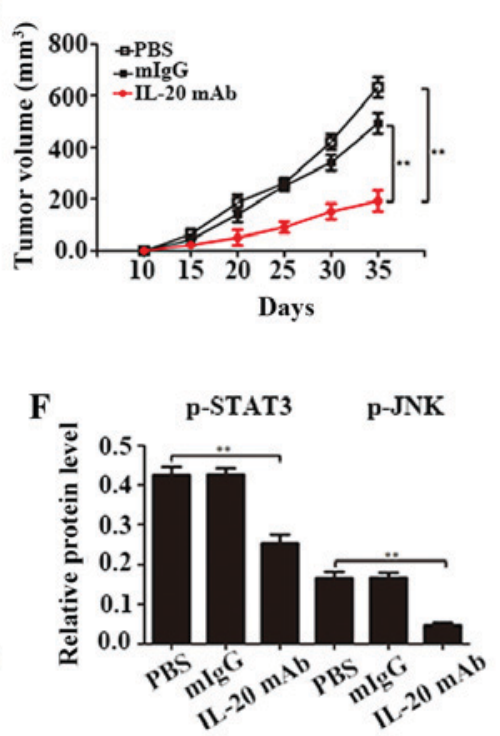

Figure 4. Effect of anti-IL-20 monoclonal antibody on tumorigenesis in nude mice. The tumor (A) size, (B) weight ("* $\mathrm{P}<0.01$ vs. PBS) and (C) volume were measured. (D) Western blot analysis of MMP-9, TGF- $\beta$, p-STAT3 and p-JNK levels in the implanted tumors. Analysis of (E) MMP-9 and TGF- $\beta$ and (F) p-STAT3 and p-JNK levels shown in panel D. ${ }^{* *} \mathrm{P}<0.01$, as indicated. $\mathrm{mIgG}$, membrane immunoglobulin G; IL, interleukin; IL-20 mAb, anti-IL-20 monoclonal antibody; p-, phosphorylated; MMP, matrix metalloproteinase; TGF, tumor growth factor; STAT, signal transducer and activator of transcription; JNK, Jun N-terminal kinase; IgG, immunoglobulin G.

or PBS. As shown in Fig. 2C and D, mIL-20 significantly enhanced Hep1-6 cell migration and invasion (both $\mathrm{P}<0.01$ vs. control), and this effect was attenuated by the anti-IL-20 mAb ( $P>0.05$ vs. control). Meanwhile, anti-IL-20 mAb suppressed Hep1-6 cell migration and invasion compared with the control group $(\mathrm{P}<0.05)$.

IL-20 regulates $H C C$ progression-related proteins in Hepl-6 cells. To determine the mechanisms underlying the IL-20-mediated promotion of tumor development, we further examined its effects on molecules related to cancer progression. As shown in Fig. 3, the levels of TGF- $\beta$ and MMP-9 were upregulated in cells to which mIL-20 had been added (Fig. 3A and C), as compared with in the control group. Moreover, phosphorylation of JNK and STAT3 was enhanced (Fig. 3B and D). These results clearly demonstrated the effects of IL-20 on factors related to HCC progression.

Blocking IL-20 with the anti-IL-20 mAb inhibits tumor growth in vivo. Considering that antibody-mediated regulation of IL-20 influenced Hep1-6 cell properties in vitro, we further analyzed whether this effect was reproducible in vivo. Overall, the tumor 
sizes and weights in the experimental group (anti-IL-20 mAb treatment) were decreased compared with those in the control group (Fig. 4A-C). Western blot data revealed that TGF- $\beta$ and MMP-9 expression, as well as the phosphorylation of STAT3 and JNK, were attenuated in the mAb-treated group (Fig. 4D-F). The collective findings indicate that the anti-IL-20 mAb effectively inhibits liver cancer cell growth, both in vivo and in vitro.

\section{Discussion}

IL-20, a member of the IL-10 family of cytokines, has been studied in various immunopathological diseases, including psoriasis, inflammatory bowel disease and rheumatoid arthritis (4). Recent studies have shown that IL-20 participates in tumor progression $(9,10)$, and is also involved in liver injury, including fibrosis and short- and long-term liver injury (12). However, the role of IL-20 in HCC is still unknown.

In the present study, we investigated the expression patterns of IL-20 and its associations with the clinicopathological characteristics of patients with HCC. The data revealed overexpression of IL-20 in liver cancer tissues. Moreover, high intratumoral IL-20 expression was significantly associated with tumor size, vessel invasion, advanced TNM stage and poor prognosis. When added into the cell culture medium, mIL-20 promoted the growth, migration and invasion of liver cancer cells in vitro, while the anti-IL-20 mAb neutralized the effect of mIL-20 in vitro, and further suppressed liver tumorigenesis in a nude mouse tumor xenograft model.

Abnormal cell proliferation and metastasis has been recognized as an important factor for tumorigenesis and cancer-related mortality (14). To further determine the mechanism underlying IL-20-induced proliferation and metastasis, expression patterns of growth, migration and invasion-related molecules were examined in Hep1-6 cells cultured under different conditions. Western blot analysis showed that IL-20 enhanced the expression of TGF- $\beta$ and MMP-9, as well as the activation of STAT3 and JNK. The anti-IL-20 mAb exerted the opposite effect, which was further verified by the in vivo data from subcutaneous tumors.

STAT3, JNK, TGF- $\beta$ and MMP-9 have been established as participants in liver tumor progression (15-17). Phosphorylated STAT3 is detected in $\sim 60 \%$ of human HCC tissues, with STAT3-positive tumors being more aggressive than STAT3-negative tumors (18). Approximately $70 \%$ of HCC tissues show positive immunostaining for phosphorylated JNK (19). JNKs are protein kinases that phosphorylate c-Jun at serine residues. c-Jun is a well-characterized oncogene, especially in liver, and its phosphorylation plays a critical role in HCC development $(20,21)$. TGF- $\beta$, when activated, targets Snail and MMP-9 to promote tumor cell invasion and migration through the epithelial-mesenchymal transition (22). Our results were consistent with these earlier findings, supporting the theory that IL-20 promotes HCC progression through regulation of TGF- $\beta$, MMP-9, STAT3 and JNK. However, the specific molecular mechanisms of this regulation are unknown. Additionally, the expression patterns and roles of IL-20 receptors in $\mathrm{HCC}$ are still unclear and require further exploration in future studies.

In conclusion, we have demonstrated for the first time that IL-20 is overexpressed in human HCC and represents a promising prognostic predictor. IL-20 promotes liver cancer growth and metastasis through enhancing the expression of TGF- $\beta$ and MMP-9, as well as the phosphorylation of STAT3 and JNK. Use of an anti-IL-20 mAb can attenuate the effects of IL-20 and effectively suppress HCC progression, both in vitro and in vivo. IL-20-specific antagonists may thus serve as potential therapeutic agents in HCC.

\section{Acknowledgements}

Not applicable.

\section{Funding}

The present study was supported by a grant from the Six Talent Peaks Project in Jiangsu Province (grant no. 2014-WSW-005).

\section{Availability of data and materials}

All data generated and/or analyzed during the present study are included in this published article.

\section{Authors' contributions}

$\mathrm{XH}, \mathrm{WD}, \mathrm{GH}$ and HJ conceived the study. WD, GH and HJ designed and performed the experiments. CZ contributed to the performance of the experiments. YJ collected the clinical samples and performed the cell culture. WD wrote the manuscript.

\section{Ethics approval and consent to participate}

Written informed consent was obtained from all patients prior to their inclusion to the study. The present study was approved by the Ethics Committee of The First Affiliated Hospital of Nanjing Medical University (Jiangsu, China).

\section{Patient consent for publication}

Written informed consent was obtained from all patients for the publication of their data.

\section{Competing interests}

The authors declare that they have no competing interests.

\section{References}

1. Venook AP, Papandreou C, Furuse J and de Guevara LL: The incidence and epidemiology of hepatocellular carcinoma: A global and regional perspective. Oncologist 154 (Suppl 4): S5-S13, 2010.

2. Chen W, Zheng R, Baade PD, Zhang S, Zeng H, Bray F, Jemal A, $\mathrm{Yu}$ XQ and He J: Cancer statistics in China, 2015. CA Cancer J Clin 66: 115-132, 2016.

3. Sabat R: IL-10 family of cytokines. Cytokine Growth Factor Rev 215: 315-324, 2010.

4. Rutz S, Wang X and Ouyang W: The IL-20 subfamily of cytokines-from host defence to tissue homeostasis. Nat Rev Immunol 14: 783-795, 2014.

5. Wegenka UM: IL-20: Biological functions mediated through two types of receptor complexes. Cytokine Growth Factor Rev 21: 353-363, 2010. 
6. Blumberg H, Conklin D, Xu WF, Grossmann A, Brender T, Carollo S, Eagan M, Foster D, Haldeman BA, Hammond A, et al: Interleukin 20: Discovery, receptor identification, and role in epidermal function. Cell 104: 9-19, 2001.

7. Wei CC, Hsu YH, Li HH, Wang YC, Hsieh MY, Chen WY, Hsing $\mathrm{CH}$ and Chang MS: IL-20: Biological functions and clinical implications. J Biomed Sci 13: 601-612, 2006.

8. Gedebjerg A, Johansen C, Kragballe K and Iversen L: IL-20, IL-21 and p40: Potential biomarkers of treatment response for ustekinumab. Acta Derm Venereol 93: 150-155, 2013.

9. Hsu YH, Hsing CH, Li CF, Chan CH, Chang MC, Yan JJ and Chang MS: Anti-IL-20 monoclonal antibody suppresses breast cancer progression and bone osteolysis in murinemodels. J Immunol 188: 1981-1991, 2012.

10. Lee SJ, Cho SC, Lee EJ, Kim S, Lee SB, Lim JH, Choi YH, Kim WJ and Moon SK: Interleukin-20 promotes migration of bladder cancer cells through extracellular signal-regulated kinase (ERK)-mediated MMP-9 protein expression leading to nuclear factor $(\mathrm{NF}-\kappa \mathrm{B})$ activation by inducing the up-regulation of p21(WAF1) protein expression. J Biol Chem 288: 5539-5552, 2013

11. Wegenka UM, Dikopoulos N, Reimann J, Adler G and Wahl C: The murine liver is a potential target organ for IL-19, IL-20 and IL-24: Type I Interferons and LPS regulatethe expression of IL-20R2. J Hepatol 46: 257-265, 2007.

12. Chiu YS, Wei CC, Lin YJ, Hsu YH and Chang MS: IL-20 and IL-20R1 antibodies protect against liver fibrosis. Hepatology 60: 1003-10014, 2014

13. Livak KJ and Schmittgen TD: Analysis of relative gene expression data using real-time quantitative PCR and the 2(-Delta Delta C(T)) method. Methods 25: 402-408, 2001.

14. Zhang Z, Zhang Y, Sun XX, Ma X and Chen ZN: Chen, microRNA-146a inhibits cancer metastasis by downregulating VEGF through dual pathways in hepatocellular carcinoma. Mol Cancer 14: 5, 2015.
15. Ji Y, Fan J, Zhang L, Tan Y, Zhou J, Zeng H, Ren Z and Xu J: Analysis of signal pathway activation in hepatocellular carcinoma: Association with clinical outcomes to sorafenib in Chinese patients. J Clin Oncol 20: 27, 2009.

16. Liu J, Chen S, Wang W, Ning BF, Chen F, Shen W, Ding J, Chen W, Xie WF and Zhang X: Cancer-associated fibroblasts promote hepatocellular carcinoma metastasis through chemokine-activated hedgehog and TGF- $\beta$ pathways. Cancer Lett 379: 49-59, 2016.

17. Lu L, Zhang Q, Wu K, Chen X, Zheng Y, Zhu C and Wu J: Hepatitis C virus NS3 protein enhances cancer cell invasion by activating matrix metalloproteinase- 9 and cyclooxygenase- 2 through ERK/p38/NF- $\mathrm{BB}$ signal cascade. Cancer Lett 356: 470-478, 2015.

18. He G, Yu GY, Temkin V, Ogata H, Kuntzen C, Sakurai T, Sieghart W, Peck-Radosavljevic M, Leffert HL and Karin M: Hepatocy te IKK $\beta / \mathrm{NF}-\kappa \mathrm{B}$ inhibits tumor promotion and progression by preventing oxidative stress-driven STAT3 activation. Cancer Cell 17: 286-297, 2010.

19. Chang Q, Zhang Y, Beezhold KJ, Bhatia D, Zhao H, Chen J, Castranova V, Shi X and Chen F: Sustained JNK1 activation is associated with altered histone $\mathrm{H} 3$ methylations in human liver cancer. J Hepatol 50: 323-333, 2009.

20. Hibi M, Lin A, Smeal T, Minden A and Karin M: Identification of an oncoprotein- and UV-responsive protein kinase that binds and potentiates the c-Jun activation domain. Genes Dev 7: 2135-2148, 1993.

21. Eferl R, Ricci R, Kenner L, Zenz R, David JP, Rath M and Wagner EF: Liver tumor development: c-Jun antagonizes the proapoptotic activity of p53. Cell 112: $181-7192,2003$

22. Giannelli G, Koudelkova P, Dituri F and Mikulits W: Role of epithelial to mesenchymal transition in hepatocellular carcinoma. J Hepatol 65: 798-808, 2016. 